\title{
Estimate Lateral Tire Force Based on Yaw Moment without Using Tire Model
}

\author{
Jun Yang, ${ }^{1}$ Wuwei Chen, ${ }^{1}$ and Yan Wang ${ }^{2}$ \\ ${ }^{1}$ School of Mechanical and Automotive Engineering, Hefei University of Technology, Hefei 230009, China \\ ${ }^{2}$ The GPS and Vehicle Dynamics Laboratory, Auburn University, AL 36849, USA \\ Correspondence should be addressed to Wuwei Chen; cww@mail.ah.hf.cn
}

Received 9 December 2013; Accepted 17 January 2014; Published 3 March 2014

Academic Editors: F. Tornabene and G.-J. Wang

Copyright (c) 2014 Jun Yang et al. This is an open access article distributed under the Creative Commons Attribution License, which permits unrestricted use, distribution, and reproduction in any medium, provided the original work is properly cited.

\begin{abstract}
This paper demonstrates the implementation of a model-based vehicle estimator, which can be used for lateral tire force estimation without using any highly nonlinear tire-road friction models. The lateral tire force estimation scheme has been designed, and it consists of the following three steps: the yaw moment estimation based on a disturbance observer, the sum of the lateral tire force of two front tires and two rear tires estimation based on a least-square method, and individual lateral tire force estimation based on a heuristic method. The proposed estimator is evaluated under two typical driving conditions and the estimation values are compared with simulator data from CarSim and experimental data provided by GM. Results to date indicate that this is an effective approach, which is considered to be of potential benefit to the automotive industry.
\end{abstract}

\section{Introduction}

To improve the handling performance and the safety of vehicles, a considerable number of active control systems for the vehicle lateral dynamics have commercially been developed and utilized over the last two decades. Lateral tireroad friction force is a vital signal that affects the stability of a vehicle under cornering. The accurate information of this force signal can greatly enhance the performance of some steering systems and active safety systems, such as electronic stability program (ESP). However, no commercial vehicles are equipped with sensors which can directly measure this force signal, which is due to either cost pressure or technical difficulty. This provides a room for an appropriate estimation algorithm. In fact, the ever-increasing demand for safety and driving comfortability makes it a very active research field in both academic society and auto industry. A vast variety of research results can be found in the literature [14]. However, tire-road friction is a very complex physical phenomenon, which is represented by various complicated mathematical models, such as Magic Formula, Fila model, and Dugoff tire model [5]. To utilize this kind of models, an online identification algorithm should be developed to detect the change of those parameters that classify road conditions. Even after considerable simplification, those models are still highly nonlinear, such as LuGre model [6], which makes the design of the controller or estimator extremely challenging. Moreover, the coupling between longitudinal and lateral tire forces further complicated the design process and lowered the robustness of the control and estimation algorithm. In this paper, we present a lateral tire force estimation algorithm without using any tire-road friction models. We also assume that the information of the longitudinal tire forces is already available. Then, the lateral tire forces of the four wheels are regarded as unknown input signals for the vehicle yaw dynamic model. A significant advantage of this approach is that no complex tire models are involved in the estimation algorithm which not only relieves the computation effort but also increases the robustness with respect to the large variation of the road conditions. Another strong point of this estimation method is that it can incorporate the longitudinal tire forces explicitly, which are often ignored in those bicycle model based estimation algorithms. Therefore, the method proposed here is particularly useful for the case where the differential braking strategy is applied. 


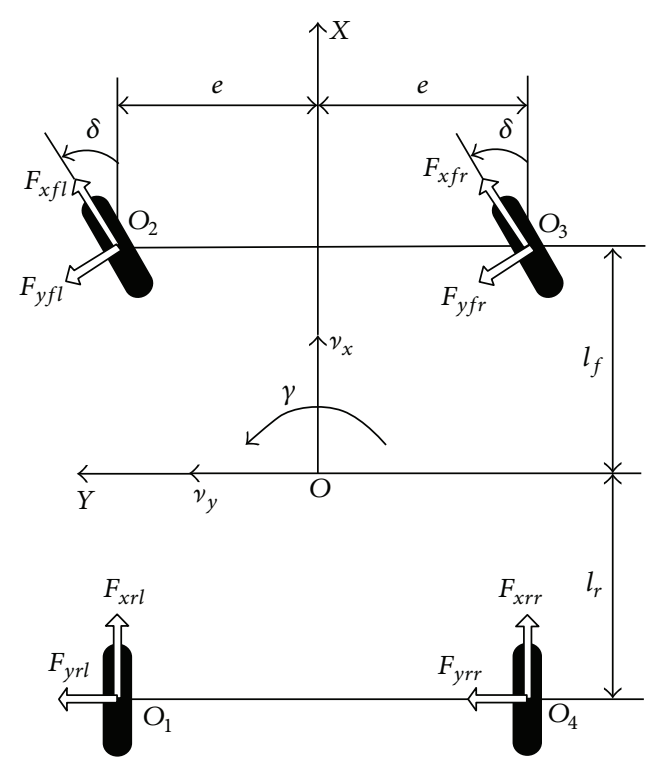

Figure 1: A 3 DOF vehicle model.

\section{Model of Automobile and Yaw Dynamic}

2.1. Dynamic Model of Automobile. A dynamic automobile model with 3 degree-of-freedom (DOF) is considered (shown in Figure 1). Take an axle perpendicular to the ground and pass through the center of mass for sprung mass as Axis $Z$, a forward direction of automobile as Axis $X$, and the center of mass as Axis $Y$. With consideration of mutual influences due to rolling, pitching, yawing, and vertical movement, the following equations of motion may be established:

$$
\begin{gathered}
m\left(v_{x}-\gamma v_{y}\right)=F_{x}, \\
m\left(\dot{v}_{y}+\gamma v_{x}\right)=F_{y}, \\
I_{z} \dot{\gamma}=M_{z},
\end{gathered}
$$

where $F_{x}$ and $F_{y}$ are the components of total tire-road friction force in the $X$ and $Y$ directions. $v_{x}$ and $v_{y}$ are the longitudinal and lateral velocities of the center of gravity (CG) of the vehicle body. $\dot{\gamma}$ represents the yaw rate along the axis perpendicular to the motion plain.

2.2. Yaw Dynamic Model. In the estimation algorithm presented in this paper, we assume that the location of the center of gravity (CG) of the vehicle is known. In what follows, we will discuss the yaw dynamic model with respect to the five different points listed here: CG $(O)$, center of the left rear wheel $\left(O_{1}\right)$, left front wheel $\left(O_{2}\right)$, right front wheel $\left(O_{3}\right)$, and right rear wheel $\left(\mathrm{O}_{4}\right)$.
(I) Yaw Dynamic w.r.t. CG: according to the rotation dynamic equation of the CG, we have

$$
\begin{gathered}
M_{o}=I_{z} \dot{\gamma}=T_{x o}+T_{y o}, \\
T_{x o}=-F_{x f l}\left(e \cos \delta-l_{f} \sin \delta\right) \\
+F_{x f r}\left(e \cos \delta+l_{f} \sin \delta\right)-e\left(F_{x l r}-F_{x r r}\right), \\
T_{y o}=F_{y f l}\left(l_{f} \cos \delta+e \sin \delta\right) \\
+F_{y f r}\left(l_{f} \cos \delta-e \sin \delta\right)-l_{r}\left(F_{y r l}+F_{y r r}\right) .
\end{gathered}
$$

(II) Yaw Dynamic w.r.t. center of the left rear wheel:

$$
M_{o 1}=I_{z} \dot{\gamma}+m a_{x} \cdot e+m a_{y} l_{r}=T_{x o 1}+T_{y o 1} .
$$

(III) Yaw Dynamic w.r.t. center of the left front wheel:

$$
M_{o 2}=I_{z} \dot{\gamma}+m a_{x} \cdot e+m a_{y} l_{f}=T_{x o 2}+T_{y o 2} .
$$

(IV) Yaw Dynamic w.r.t. center of the right front wheel:

$$
M_{o 3}=I_{z} \dot{\gamma}-m a_{x} \cdot e-m a_{y} l_{f}=T_{x o 3}+T_{y o 3} .
$$

(V) Yaw Dynamic w.r.t. center of the right rear wheel:

$$
M_{o 4}=I_{z} \dot{\gamma}-m a_{x} \cdot e+m a_{y} l_{r}=T_{x o 4}+T_{y o 4} .
$$

In the above, we derived the yaw dynamic model of the vehicle body w.r.t. five different reference points. Here, we lump them together as a matrix form as follows:

$$
\begin{aligned}
& T_{x}=G \cdot F_{x}, \\
& T_{y}=H \cdot F_{y},
\end{aligned}
$$

where

$$
\begin{aligned}
& T_{x}=\left[\begin{array}{lllll}
T_{x o} & T_{x o 1} & T_{x o 2} & T_{x o 3} & T_{x o 4}
\end{array}\right]^{T}, \\
& T_{y}=\left[\begin{array}{lllll}
T_{y o} & T_{y o 1} & T_{y o 2} & T_{y o 3} & T_{y o 4}
\end{array}\right]^{T}, \\
& F_{x}=\left[\begin{array}{llll}
F_{x f l} & F_{x f r} & F_{x r l} & F_{x r r}
\end{array}\right]^{T}, \\
& F_{y}=\left[\begin{array}{lll}
F_{y f l} & F_{y f r} & F_{y r l}+F_{y r r}
\end{array}\right]^{T} \text {, } \\
& G=\left[\begin{array}{cccc}
-e \cos \delta+l_{f} \sin \delta & e \cos \delta+l_{f} \sin \delta & -e & e \\
\left(l_{f}+l_{r}\right) \sin \delta & 2 e \cos \delta+\left(l_{f}+l_{r}\right) \sin \delta & 0 & 2 e \\
0 & 2 e \cos \delta & 0 & 2 e \\
-2 e \cos \delta & 0 & -2 e & 0 \\
-2 e \cos \delta+\left(l_{f}+l_{r}\right) \sin \delta & \left(l_{f}+l_{r}\right) \sin \delta & -2 e & 0
\end{array}\right], \\
& H=\left[\begin{array}{ccc}
l_{f} \cos \delta+e \sin \delta & l_{f} \cos \delta-e \sin \delta & -l_{r} \\
\left(l_{f}+l_{r}\right) \cos \delta & \left(l_{f}+l_{r}\right) \cos \delta-2 e \sin \delta & 0 \\
0 & -2 e \sin \delta & -\left(l_{f}+l_{r}\right) \\
2 e \sin \delta & 0 & -\left(l_{f}+l_{r}\right) \\
\left(l_{f}+l_{r}\right) \cos \delta+2 e \sin \delta & \left(l_{f}+l_{r}\right) \cos \delta & 0
\end{array}\right]
\end{aligned}
$$




\section{Estimate Moment of Lateral Tire Force Based on Disturbance Observer}

In this section, we will discuss how to combine the yaw dynamic model of the vehicle body, yaw rate, and acceleration in longitudinal and lateral directions to estimate the moment of the lateral tire forces w.r.t. different points in the vehicle body. Here, we will assume that the information of location of the center of gravity of the vehicle and the longitudinal force of each tire are both available. The general structure of the disturbance observer is shown in Figure 2 which is included by a closed green dash line.

As shown in Figure 2, the disturbance observer incorporates a virtual yaw dynamic model, which is composed of the yaw dynamical equations w.r.t. five different reference points and a feedback controller. The virtual yaw dynamic model is driven by the torque $T_{x}$. The real yaw dynamics of the vehicle body is driven by both the torque produced by the longitudinal and lateral tire forces. The controller's input is the tracking error of the yaw rate, which is indeed the difference between the measured yaw rate and the virtual model's output. The design of the controller can resort to those general feedback control design methods that aim at achieving good tracking performance. In this paper, we use the PID controller as an example. If the virtual model's output can follow the measured yaw rate closely enough, it implies that the controller's output or the control action indeed compensates the unmeasured input, which is the torque produced by the lateral tire force in this case.

For illustrating the proposed estimator clearly, we will take the yaw dynamic model w.r.t. CG as an example to analyze it. From (2), the longitudinal tire forces of all the four wheels are known; the torque $T_{x o}$ can be directly calculated. Obviously, the disturbance observer can be used to estimate $T_{y o}$ based on the measured yaw rate signal $\dot{\gamma}$. The derivation of the estimation of $T_{y o}$ is shown in the following [7]:

$$
I_{z} \dot{\gamma}=T_{x o}+T_{y o}
$$

$\Downarrow$ Laplace Transformation

$$
I_{z} s \gamma(s)=T_{x o}(s)+T_{y o}(s)
$$

$\Downarrow$ Low Pass Filter

$$
\frac{I_{z} s}{T_{s}+1} \gamma(s)=\frac{1}{T_{s}+1}\left[T_{x o}(s)+T_{y o}(s)\right] \text {. }
$$

Then, we formulate the observer's state equation in $s$ domain as

$$
\begin{aligned}
\frac{I_{z} s}{T_{s}+1} \widehat{\gamma}(s)= & \frac{1}{T_{s}+1}\left[T_{x o}(s)+\bar{T}_{y o}(s)\right]+K_{p} \tilde{\gamma}(s) \\
& +K_{i} \frac{1}{s} \widetilde{\gamma}(s)+\frac{-I_{z}+K_{d}}{T_{s}+1} s \tilde{\gamma}(s),
\end{aligned}
$$

where $\widehat{\gamma}(s)$ is the Laplace transform of the estimation of yaw rate $\gamma . T_{x o}$ can be regarded as the input to the observer. $\bar{T}_{y o}$ is an initial guess or the nominal value of the moment of lateral tire force. $\tilde{\gamma}=\gamma-\widehat{\gamma}$ is defined as the deviation between the measured yaw rate and its estimation. The tail part of (11) is composed of three feedback correction items. If we subtract this equation from the third formula in (10), the following formula shows the relation between yaw rate error $\tilde{\gamma}$ and the deviation between the unmeasured torque signals $T_{y_{0}}$ and $\bar{T}_{y o}$ :

$$
\begin{aligned}
-\frac{1}{T_{s}+1}\left[T_{y o}(s)-\bar{T}_{y o}(s)\right]= & K_{p} \tilde{\gamma}(s)+K_{i} \frac{1}{s} \tilde{\gamma}(s) \\
& +\frac{K_{d}}{T_{s}+1} s \tilde{\gamma}(s) .
\end{aligned}
$$

Based on (12), we can estimate the torque produced by the lateral tire force as follows:

$$
\begin{aligned}
\widehat{T}_{y o}(s) & =\bar{T}_{y o}(s)+\frac{1}{T_{s}+1} \widetilde{T}_{y o}(s) \\
& =\bar{T}_{y o}(s)-\left[K_{p} \widetilde{\gamma}(s)+K_{i} \frac{1}{s} \widetilde{\gamma}(s)+\frac{K_{d}}{T_{s}+1} s \widetilde{\gamma}(s)\right],
\end{aligned}
$$

where $\widehat{T}_{y o}$ is the Laplace transformation of estimated friction force $T_{y o}$. While $\widetilde{T}_{y o}=T_{y o}-\widehat{T}_{y o}$ is defined as the deviation between the real torque signal and its estimation. It is easy to find that the right side of (11) is the transfer function of a PID controller. Four tuning parameters, which are $K_{p}, K_{i}, K_{d}$, and $T$ in the feedback PID controller, aim at making the virtual inertia model's output signal $\widehat{\gamma}$ track the measure yaw rate signal $\gamma$ as closely as possible. Therefore, the controller's outputs actually compensate the difference between the unmeasured input signal, which is $T_{y o}$ for this case, and its initial guess $\bar{T}_{y o}$. Moreover, the choice of $\bar{T}_{y_{o}}$ is quite flexible. But the more close to $T_{y o}$ it is, the less control effort is needed. Here, we choose it as a constant zero all the time just for simplicity.

The low pass weighted filter $1 /\left(T_{s}+1\right)$ gives us the approximation of the torque signal in the low frequency band. It is obvious that the smaller the time constant $T$ is, the wider the frequency band of the friction force deviation information can be exacted from (12). But it is not wise to include very high frequency components of $\widehat{T}_{y o}$, because we need to utilize this filter to suppress the noise in the deviation signal $\tilde{\gamma}$ and approximate the differentiator in the PID controller in low frequency domain.

\section{Lateral Force Estimation}

In Section 3, we have discussed using the disturbance observer to estimate the moment of the lateral tire forces w.r.t. five different reference points on the vehicle body. In this section, we will study how to exact the lateral force acting on the individual tire from the existing estimated torque signals.

As shown in (2), the lateral tire forces can be transferred to their moments through a steering angle dependent matrix $H$. Therefore, the linear mapping between $T_{y}$ and $F_{y}$ provides an approach to estimate the components in $F_{y}$ from the existing estimated torque signals $\widehat{T}_{y}$ by using the least square 


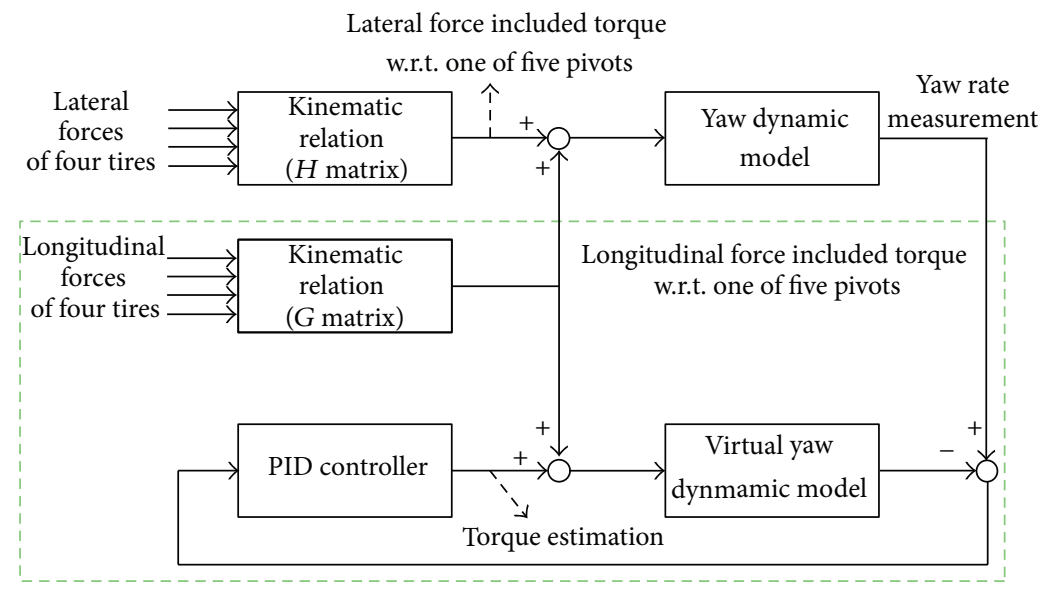

FIGURE 2: General structure of the disturbance observer based torque estimation.

method, which aims at finding a vector $\widehat{F}_{y}$ that minimizes the following quadratic cost function [8]:

$$
\min _{\widehat{F}_{y}}\left\|\widehat{T}_{y}-H \widehat{F}_{y}\right\|_{2}
$$

where $\widehat{T}_{y}$ can be written as

$$
\widehat{T}_{y}=H \cdot F_{y}+\zeta
$$

$\zeta$ represents the torque estimation error, which may come from the inaccurate model parameters, such as mass and yaw inertia, and inaccurate longitudinal tire forces. The analytic solution (if it exists) of the unconstrained least square problem is

$$
\widehat{F}_{y}=\left(H^{T} H\right)^{-1} H^{T} \widehat{T}_{y},
$$

where $\widehat{F}_{y}$ is the estimation of $F_{y}$ from the estimated torque moment $\widehat{T}_{y}$. If the vector signal $\zeta$, which results from model uncertainty, is composed of zero-mean stochastic signals, the solution of the least square problem will give us the robust estimation of $F_{y}$. However, the necessary and sufficient condition for the existence of a unique solution of the global minimum of the quadratic cost function shown in (15) is that the matrix $H$ should have full column rank, which is equal to 3 in our case. This is equivalent to the condition that the matrix $H^{T} H$ is invertible.

Unfortunately, the matrix $H$ does not satisfy the full column condition in case the steering angle $\delta$ is equal to zero. A brief proof is shown as follows:

$$
\begin{aligned}
\sin \delta & =0 \Longrightarrow H \\
& =\left[\begin{array}{ccc}
l_{f} \cos \delta & l_{f} \cos \delta & -l_{r} \\
\left(l_{f}+l_{r}\right) \cos \delta & \left(l_{f}+l_{r}\right) \cos \delta & 0 \\
0 & 0 & -\left(l_{f}+l_{r}\right) \\
0 & 0 & -\left(l_{f}+l_{r}\right) \\
\left(l_{f}+l_{r}\right) \cos \delta & \left(l_{f}+l_{r}\right) \cos \delta & 0
\end{array}\right] .
\end{aligned}
$$

It is easy to find that the first and second columns of the matrix $H$ are the same. This implies that there are only 2 independent columns in the matrix $H$ or it is equivalent to say that the matrix $H$ loses one column rank. It is also understandable that the matrix $H$ will be closed to rank deficiency if the steering angles are small enough to make the trigonometry approximation shown in (16) valid. Although it is just the case that the matrix $H$ is closed to losing rank but not exactly, it will still cause numerical troubles when the computer does the matrix inverse operation. Moreover, the small steering angle is the case for most steering operations of a vehicle, such as double or single lane change.

To overcome the rank deficiency problem discussed above, the kinematic model in (2) should be modified in an approximation way. Firstly, we assume that the sinusoid function of steering angle can be treated as zero approximately. Then, we will analyze the kinematic relation between the lateral tire forces and its moment w.r.t. different reference points individually.

(i) For reference point $O$ : the assumptions stated allow us to rewrite (2)-(6) as:

$$
T_{y o} \approx\left(F_{y f l}+F_{y f r}\right) l_{f} \cos \delta-l_{r}\left(F_{y r l}+F_{y r r}\right) .
$$

(ii) For reference point $\mathrm{Ol}$ :

$$
T_{y o 1} \approx\left(F_{y f l}+F_{y f r}\right)\left(l_{f}+l_{r}\right) \cos \delta .
$$

(iii) For reference point $\mathrm{O} 2$ :

$$
T_{y o 2} \approx-\left(F_{y r l}+F_{y r r}\right)\left(l_{f}+l_{r}\right) .
$$

(iv) For reference point $\mathrm{O3}$ :

$$
T_{y o 3} \approx-\left(F_{y r l}+F_{y r r}\right)\left(l_{f}+l_{r}\right) .
$$

(v) For reference point 04 :

$$
T_{y o 4} \approx\left(F_{y f l}+F_{y f r}\right)\left(l_{f}+l_{r}\right) \cos \delta .
$$


From the above simplified kinematic models, we can derive the following equations for $T_{y o 1}-T_{y o 2}$ and $T_{y o 4}-T_{y o 3}$ :

$$
\begin{aligned}
T_{y o 1}-T_{y o 2}= & \left(F_{y f l}+F_{y f r}\right)\left(l_{f}+l_{r}\right) \cos \delta \\
& +\left(F_{y r l}+F_{y r r}\right)\left(l_{f}+l_{r}\right), \\
T_{y o 4}-T_{y o 3}= & \left(F_{y f l}+F_{y f r}\right)\left(l_{f}+l_{r}\right) \cos \delta \\
& +\left(F_{y r l}+F_{y r r}\right)\left(l_{f}+l_{r}\right) .
\end{aligned}
$$

It is clearly that the two equations are the same on the right side shown in (22) and (23). Therefore, they can be combined together as follows:

$$
\begin{aligned}
& \frac{\left(T_{y o 1}-T_{y o 2}\right)+\left(T_{y o 4}-T_{y o 3}\right)}{2} \\
& =\left(F_{y f l}+F_{y f r}\right)\left(l_{f}+l_{r}\right) \cos \delta \\
& +\left(F_{y r l}+F_{y r r}\right)\left(l_{f}+l_{r}\right) \text {. }
\end{aligned}
$$

Finally, we can combine together (17)-(24) to form a matrix form as

$$
T_{y 1}=H_{1} \cdot F_{y 1}
$$

where

$$
\begin{gathered}
T_{y 1}=\left[T_{y o} \frac{\left(T_{y o 1}-T_{y o 2}\right)+\left(T_{y o 4}-T_{y o 3}\right)}{2}\right]^{T}, \\
H_{1}=\left[\begin{array}{cc}
l_{f} \cos \delta & -l_{r} \\
\left(l_{f}+l_{r}\right) \cos \delta & l_{f}+l_{r}
\end{array}\right]^{T}, \\
F_{y 1}=\left[\begin{array}{ll}
F_{y f l}+F_{y f r} & F_{y r l}+F_{y r r}
\end{array}\right]^{T} .
\end{gathered}
$$

Compared with the matrix $H$, the new matrix $H_{1}$ will not meet the rank deficiency problem even in case of small steering angle. It has full column rank and the solution of the least square problem shown below will give us the best estimate of the vector $F_{y 1}$ :

$$
\widehat{F}_{y 1}=\left(H_{1}^{T} H_{1}\right)^{-1} H_{1}^{T} T_{y 1} \text {. }
$$

However, the cost is that only the sum of the lateral tire forces of the two front wheels $F_{y f l}+F_{y f r}$ and the two rear wheels $F_{y r l}+F_{y r r}$ can be estimated rather than the individual tire force. Obviously, additional constraints are needed to estimate the four lateral tire forces. Here, we will use the normal load information and a heuristic lookup table to estimate individual tire force from the existing cumulative values $F_{y f l}+F_{y f r}$ and $F_{y r l}+F_{y r r}$. The relationship between normal load and the lateral force of each tire can be approximated as a linear relation listed as follows:

$$
\frac{F_{y f l}}{F_{z f l}} \approx \frac{F_{y f r}}{F_{z f r}}, \quad \frac{F_{y r l}}{F_{z r l}} \approx \frac{F_{y r r}}{F_{z r r}},
$$

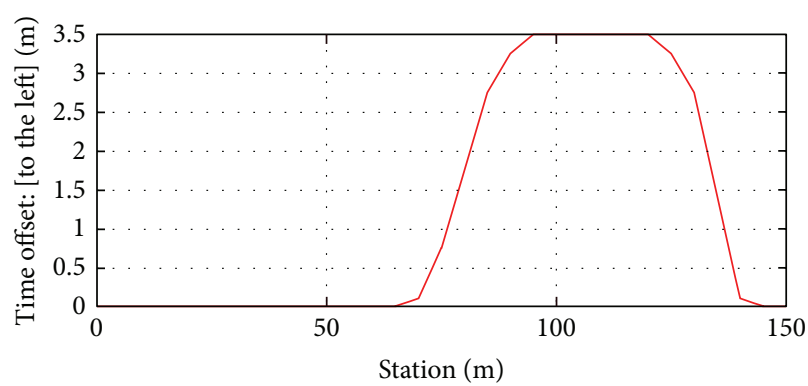

Figure 3: Double lane change: motion profile.

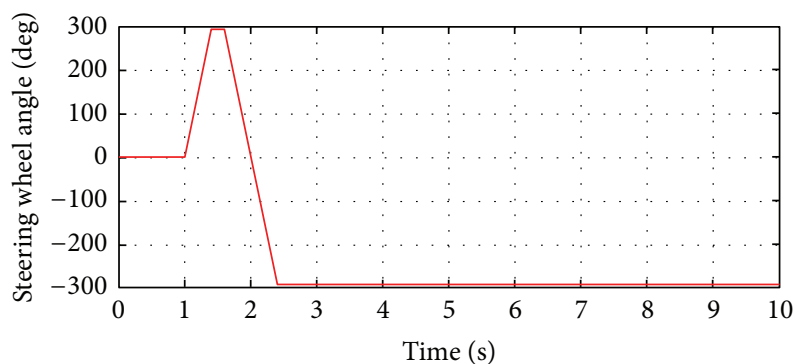

FIGURE 4: Fish hook: profile of steering angle.

where $F_{z f l}, F_{z f r}, F_{z r l}$, and $F_{z r r}$ denote the normal load of the corresponding tires. For providing accurate results compared with both the simulation data from CarSim and experimental data, a simple and heuristic method to improve the estimation accuracy is to add a lateral acceleration dependent correction term to capture the nonlinear relation between the lateral force and the normal load:

$$
\begin{aligned}
& \widehat{F}_{y f l} \approx\left(\frac{F_{z f l}}{F_{z f l}+F_{z f r}}+\lambda a_{y}\right)\left(F_{y f l}+F_{y f r}\right), \\
& \widehat{F}_{y f r} \approx\left(\frac{F_{z f r}}{F_{z f l}+F_{z f r}}-\lambda a_{y}\right)\left(F_{y f l}+F_{y f r}\right),
\end{aligned}
$$

where $a_{y}$ is the lateral acceleration of CG. $\lambda$ is a weighting factor to correct the relation shown in (28) from linear to nonlinear. The lateral tire forces $F_{y r l}$ and $F_{y r r}$ can be estimated just like (29) which are omitted here.

\section{Simulation Results}

To verify the estimation algorithm for the lateral tire forces discussed in this paper, CarSim data and experimental data provided by GM are utilized. Then, two typical experiments are selected for CarSim simulation: first of which is the standard double lane change shown in Figure 3 and second of which is fish hook shown in Figure 4. The vehicle test was done by GM and then GM provided the experimental data for this study.

5.1. CarSim Data. A big pickup with the gross vehicle weight of $2024 \mathrm{~kg}$ and the yaw inertia of $3200 \mathrm{~kg} \cdot \mathrm{m}^{2}$ has been used for the simulation studies. Meanwhile, $l_{f}=1.070 \mathrm{~m}$, 


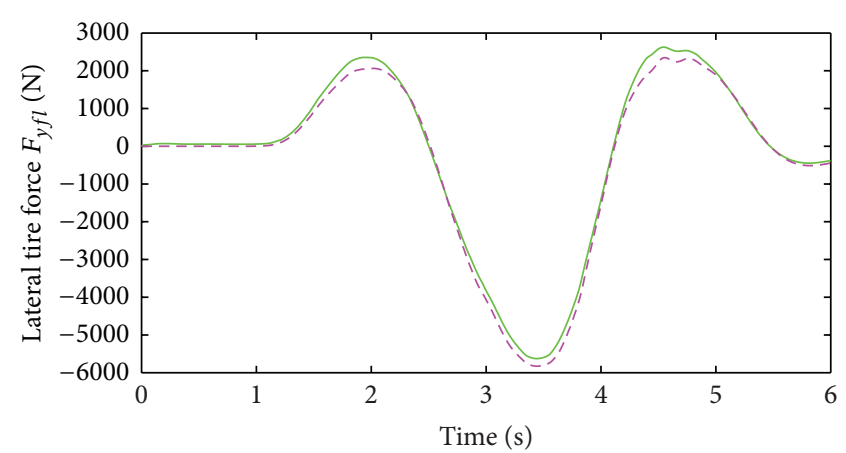

(a) Lateral force for front-left tire

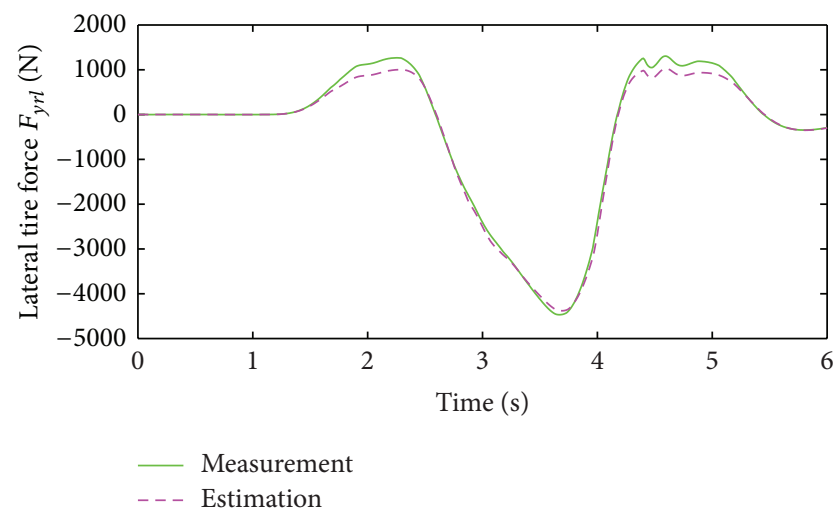

(c) Lateral force for rear-left tire

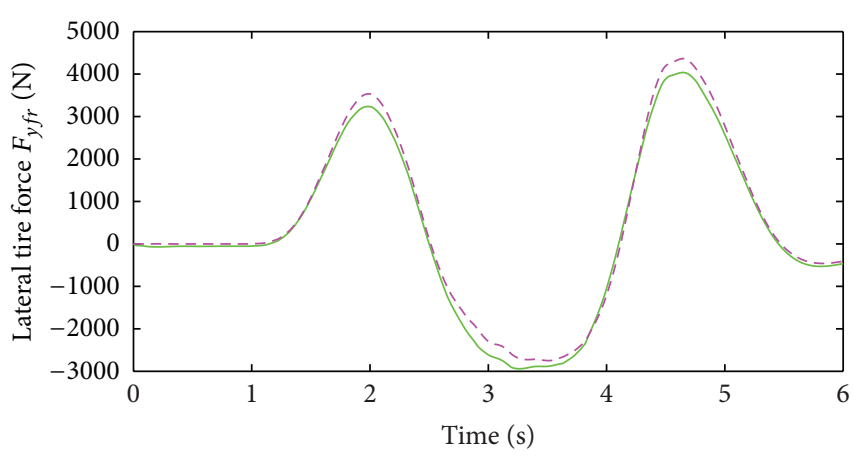

(b) Lateral force for front-right tire

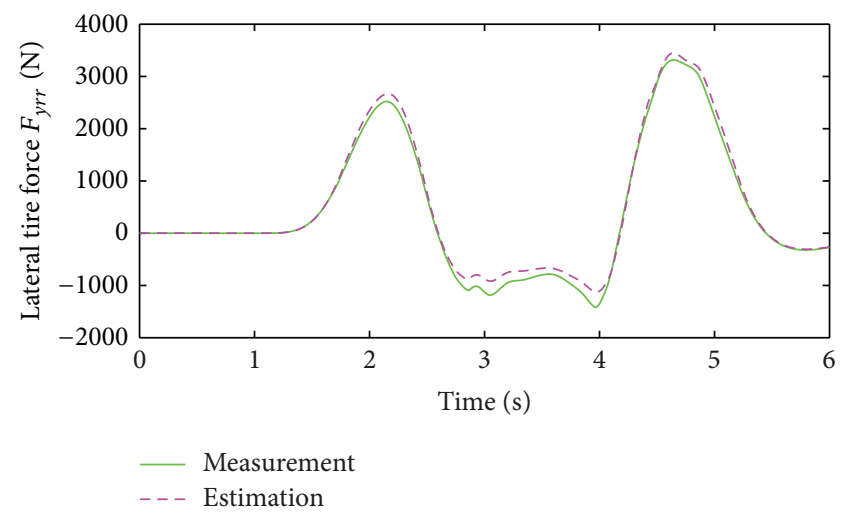

(d) Lateral force for rear-right tire

FIgURE 5: CarSim data for double lane change with $\mu=0.8$ at four tires.

$l_{r}=1.953 \mathrm{~m}$, and $e=0.871 \mathrm{~m}$. The simulation results for the two cases are shown in Figures 5, 6, and 7.

Figure 5 shows the simulation results with double lane change front steering angle input in which road coefficient $\mu$ is 0.8 and Figure 6 shows the road coefficient $\mu=0.2$. As shown in Figures 5-6, the proposed tire force estimator shows good performance.

For fish hook condition, Figure 7 shows lateral forces with the estimates and measured data compared. It is clear that estimate of lateral forces match the measured data excellently. It also can be shown that estimates of lateral forces capture the trend in actual measured data. In addition, the car loses stability when the coefficient of road $\mu$ is 0.2 for this situation.

5.2. Experimental Data. In this section, experimental evaluations are carried out to verify the effectiveness of the proposed estimated method. The experimental data is provided by GM. The vehicle parameters used in this experimental test are shown in Table 1.

With maximum $\mu$ is 0.8 , the experimental results for the lateral tire forces can be seen in Figure 8. From these experimental results, we can easily see that the estimated lateral tire force can follow the measured data very well; these results demonstrate that the proposed estimator is effective.
TABle 1: Vehicle parameters.

\begin{tabular}{lc}
\hline Parameters & Values \\
\hline Vehicle total mass $\mathrm{m} / \mathrm{kg}$ & 2083 \\
Vehicle yaw moment of inertia $I_{z} /\left(\mathrm{kg} \cdot \mathrm{m}^{2}\right)$ & 3100 \\
$\begin{array}{l}\text { Distance from vehicle COG to front axle } \\
\text { and rear axle }\left(l_{f}, l_{r}\right) / \mathrm{m}\end{array}$ & $1.325,1.637$ \\
Front and rear track width $e /(\mathrm{m})$ & 0.78 \\
\hline
\end{tabular}

\section{Conclusions}

In this paper, we proposed a model-based algorithm to estimate the lateral tire force without resorting to complex tire-road friction model. A significant advantage of this approach is that no complex tire models are involved in the estimation algorithm which not only relieves the computation effort but also increases the robustness with respect to the large variation of the road conditions. Another strong point of this estimation method is that it can incorporate the longitudinal tire forces explicitly, which are often ignored in those bicycle model based estimation algorithm. The CarSim simulation and experimental results demonstrate the ability of this approach to provide accurate estimations and show its 


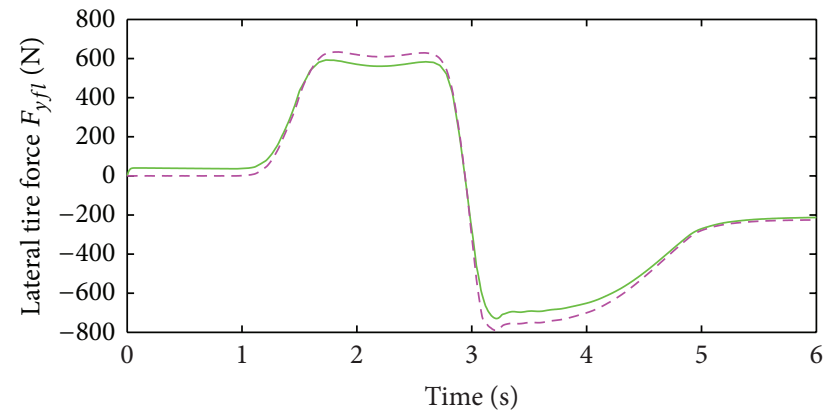

(a) Lateral force for front-left tire

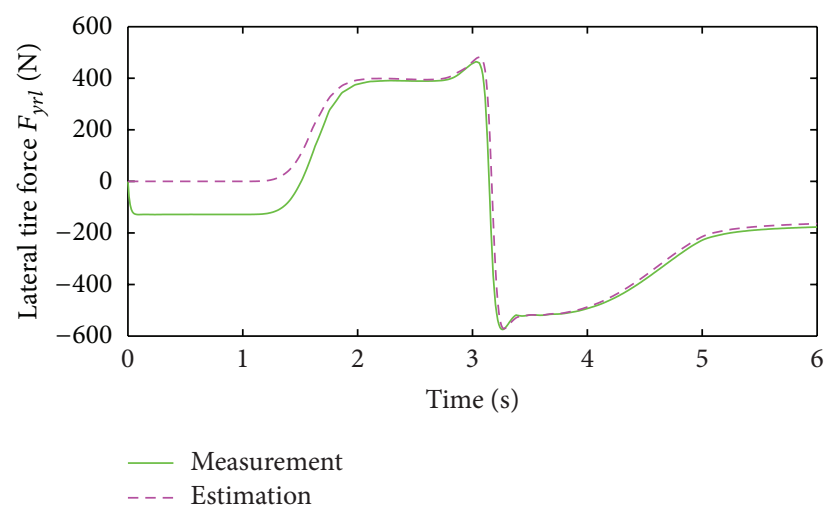

(c) Lateral force for rear-left tire

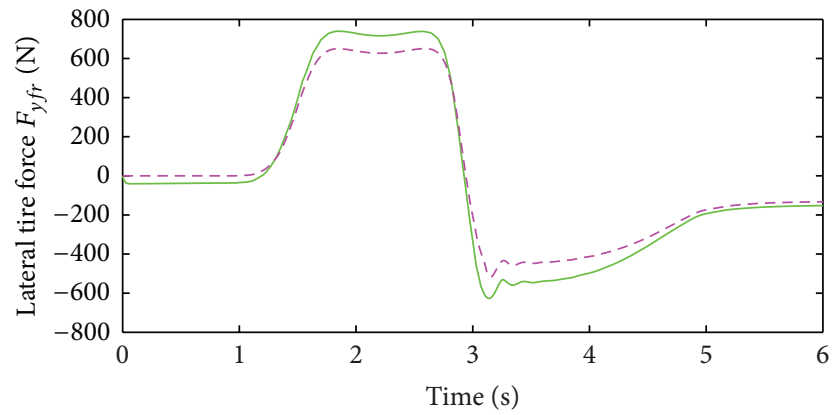

(b) Lateral force for front-right tire

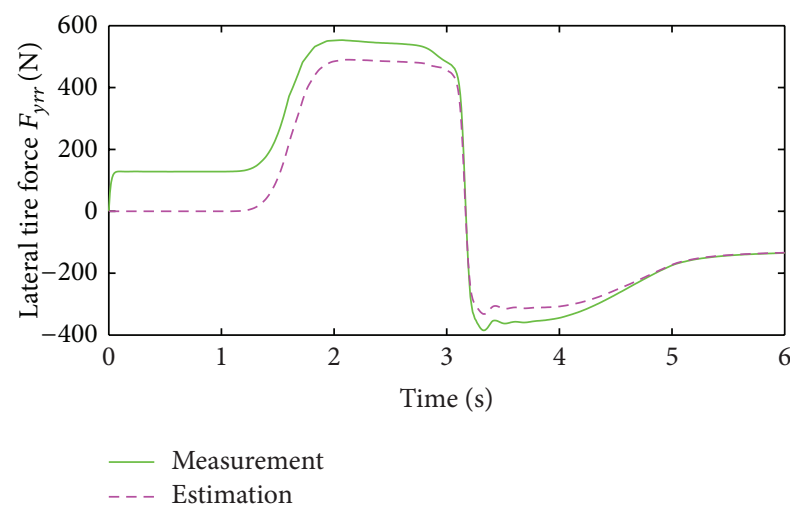

(d) Lateral force for rear-right tire

FIgURE 6: CarSim data for double lane change with $\mu=0.2$ at four tires.

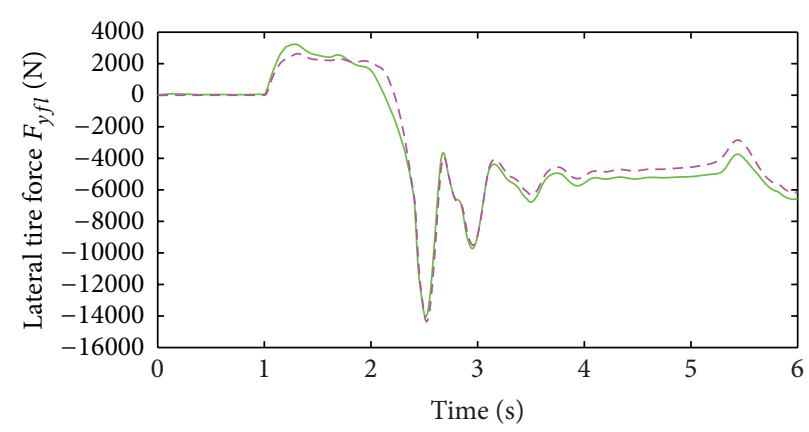

(a) Lateral force for front-left tire

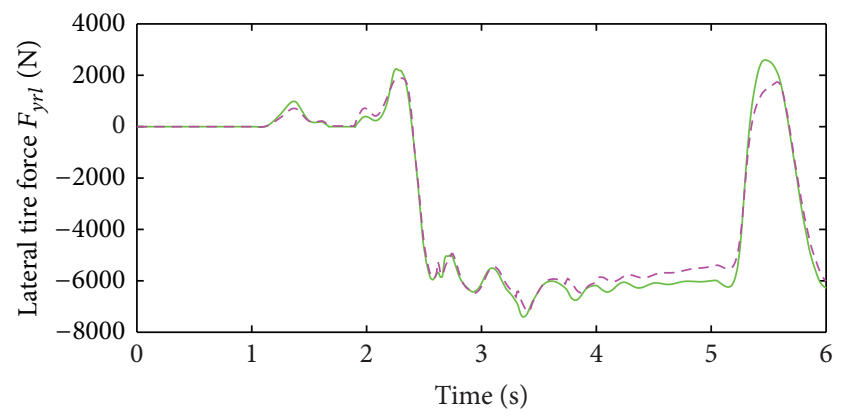

- Measurement

(c) Lateral force for rear-left tire

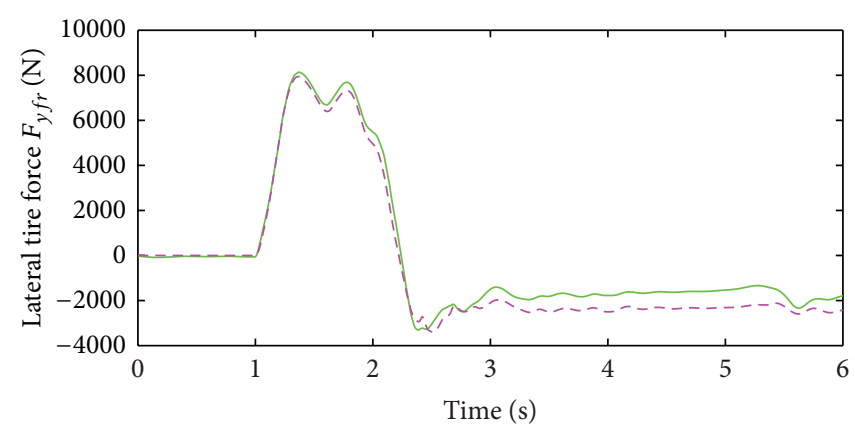

(b) Lateral force for front-right tire

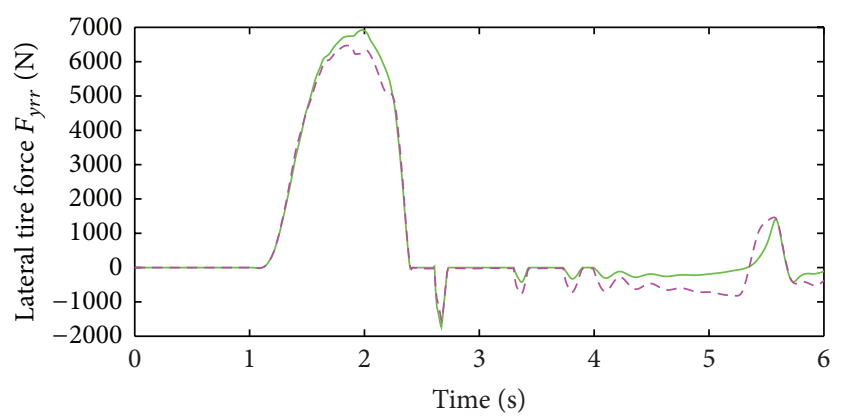

— Measurement

- - - Estimation

(d) Lateral force for rear-right tire

Figure 7: CarSim data for fish hook with $\mu=0.8$ at four tires. 


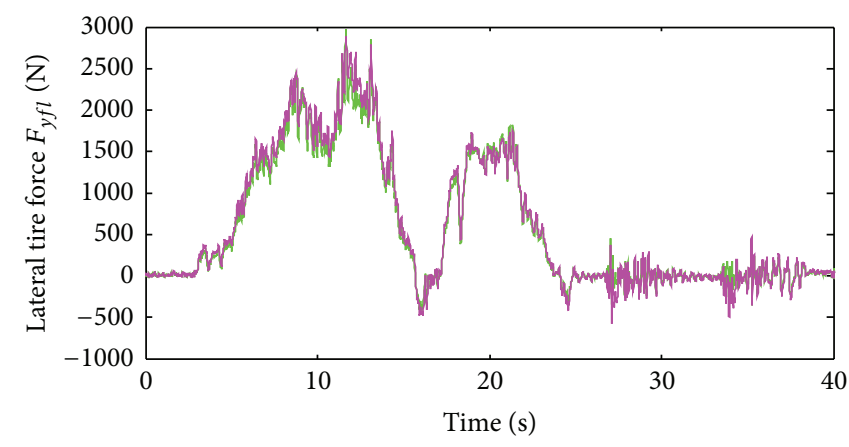

(a) Lateral force for front-left tire

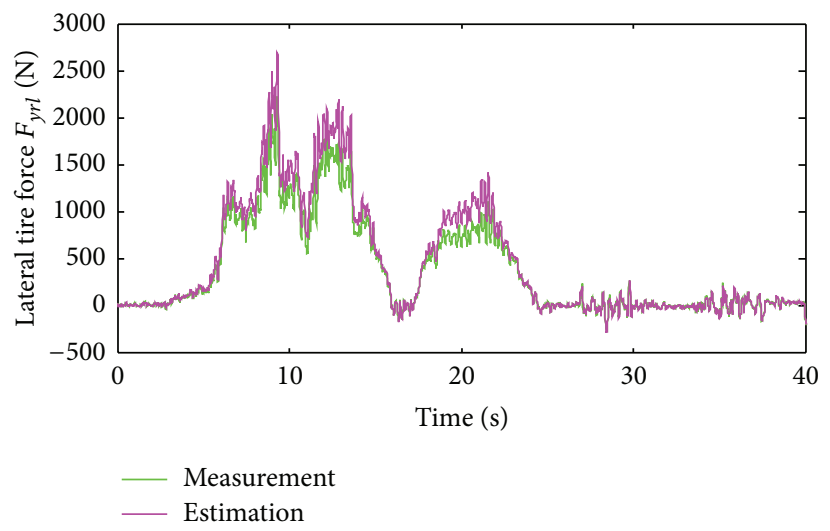

(c) Lateral force for rear-left tire

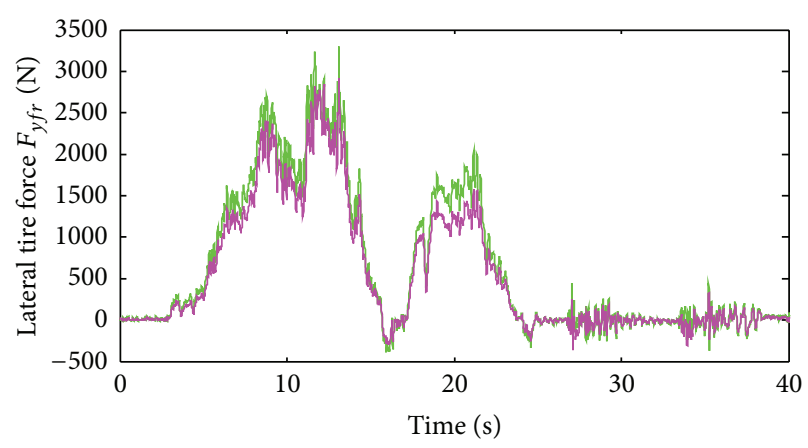

(b) Lateral force for front-right tire

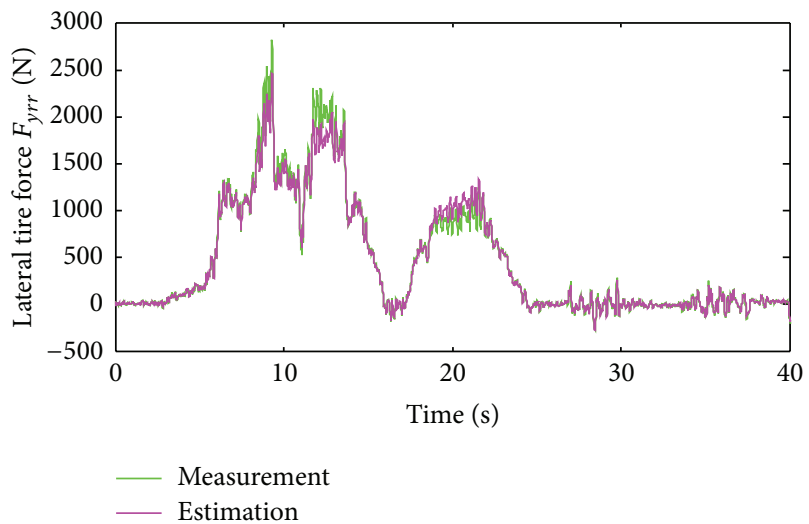

(d) Lateral force for rear-right tire

FIGURE 8: Experimental results provided by GM for four lateral tire forces.

practical potential as a low-cost solution for calculating lateral tire forces.

\section{Conflict of Interests}

The authors declare that there is no conflict of interests regarding the publication of this paper.

\section{Acknowledgments}

This project is supported by National Natural Science Foundation of China (Grant nos. 51075112 and 51175135), the GPS and Vehicle Dynamics Laboratory at Auburn University in Alabama, USA, and GM (General Motors).

\section{References}

[1] L. R. Ray, "Nonlinear tire force estimation and road friction identification: simulation and experiments," Automatica, vol. 33, no. 10, pp. 117-124, 1995.

[2] M. A. Wilkin, D. C. Crolla, M. C. Levesley, and W. J. Manning, "Design of a robust tyre force estimator using an extended Kalman filter," Tech. Rep. 2005-01-0400, SAE, Warrendale, Pa, USA, 2005.

[3] F. Gustafsson, "Statistical signal processing for automotive safety systems," in Proceedings of the IEEE/SP 13th Workshop on Statistical Signal Processing, pp. 1428-1435, July 2005.
[4] H. Shraim, B. Ananou, L. Fridman, H. Noura, and M. Ouladsine, "Sliding mode observers for the estimation of vehicle parameters, forces and states of the center of gravity," in Proceedings of the 45th IEEE Conference on Decision and Control (CDC '06), pp. 1635-1640, San Diego, Calif, USA, December 2006.

[5] R. Rajamani, Vehicle Dynamics and Control, Springer, New York, NY, USA, 2005.

[6] E. Velenis, P. Tsiotras, C. Canudas-De-Wit, and M. Sorine, "Dynamic tyre friction models for combined longitudinal and lateral vehicle motion," Vehicle System Dynamics, vol. 43, no. 1, pp. 3-29, 2005.

[7] R. N. Jazar, Vehicle Dynamics: Theory and Applications, Springer, 1st edition, 2008.

[8] T. Kailath, A. H. Sayed, and B. Hassibi, Linear Estimation, Prentice Hall, 1st edition, 2000. 

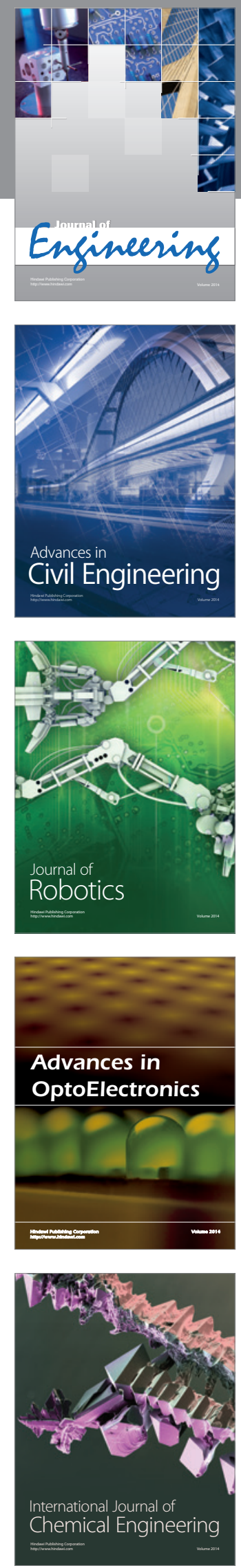

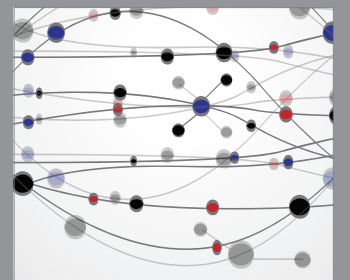

The Scientific World Journal
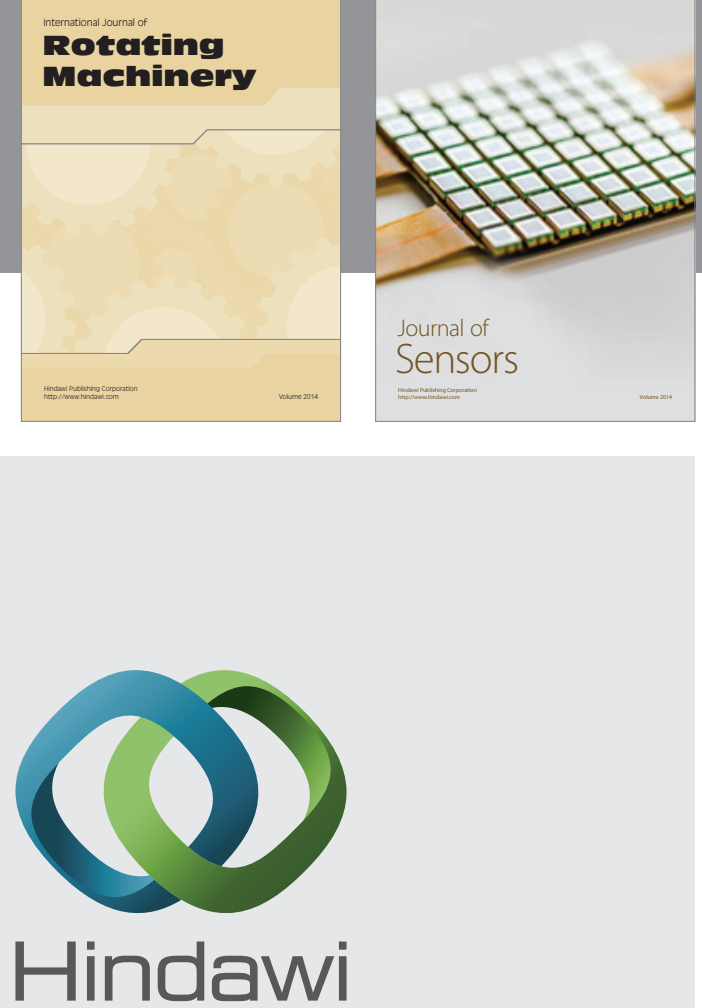

Submit your manuscripts at http://www.hindawi.com
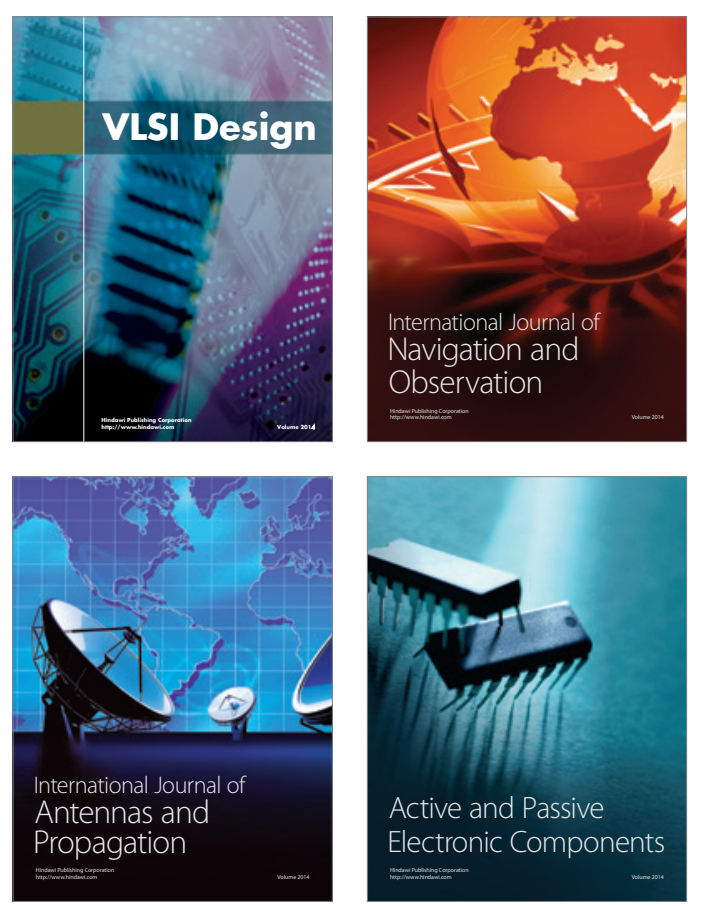
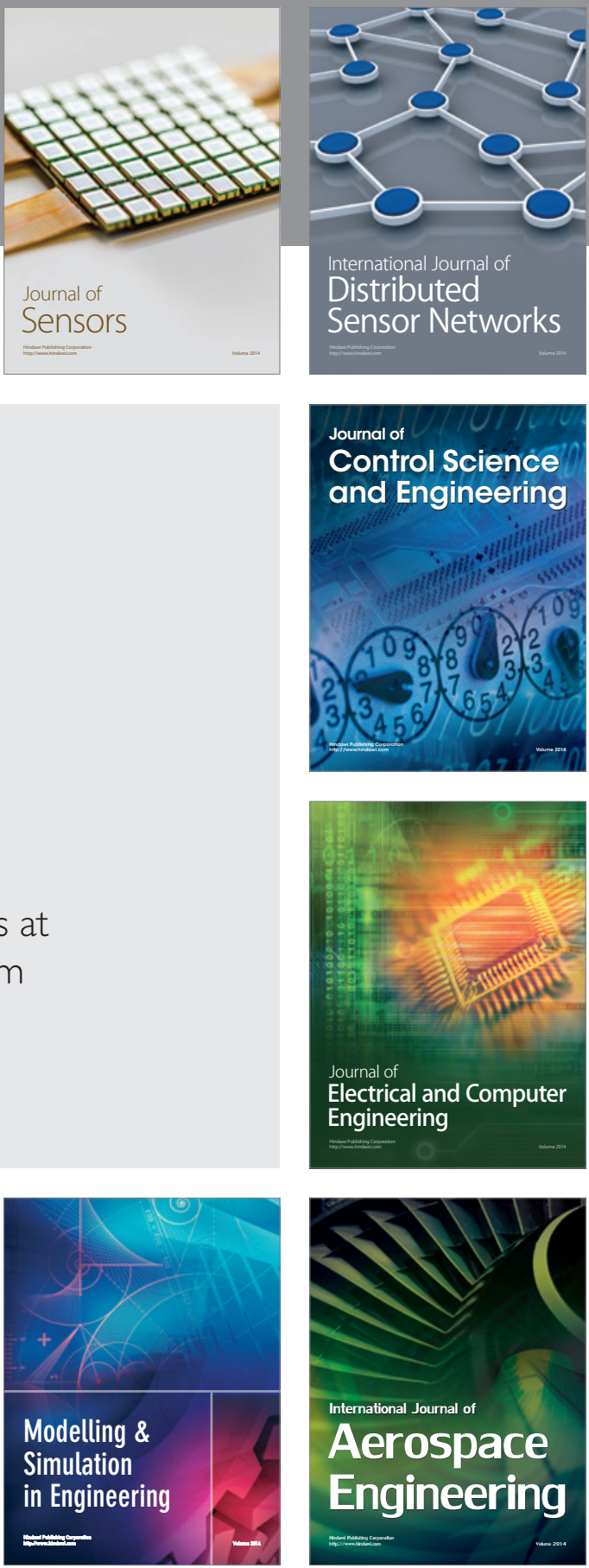

Journal of

Control Science

and Engineering
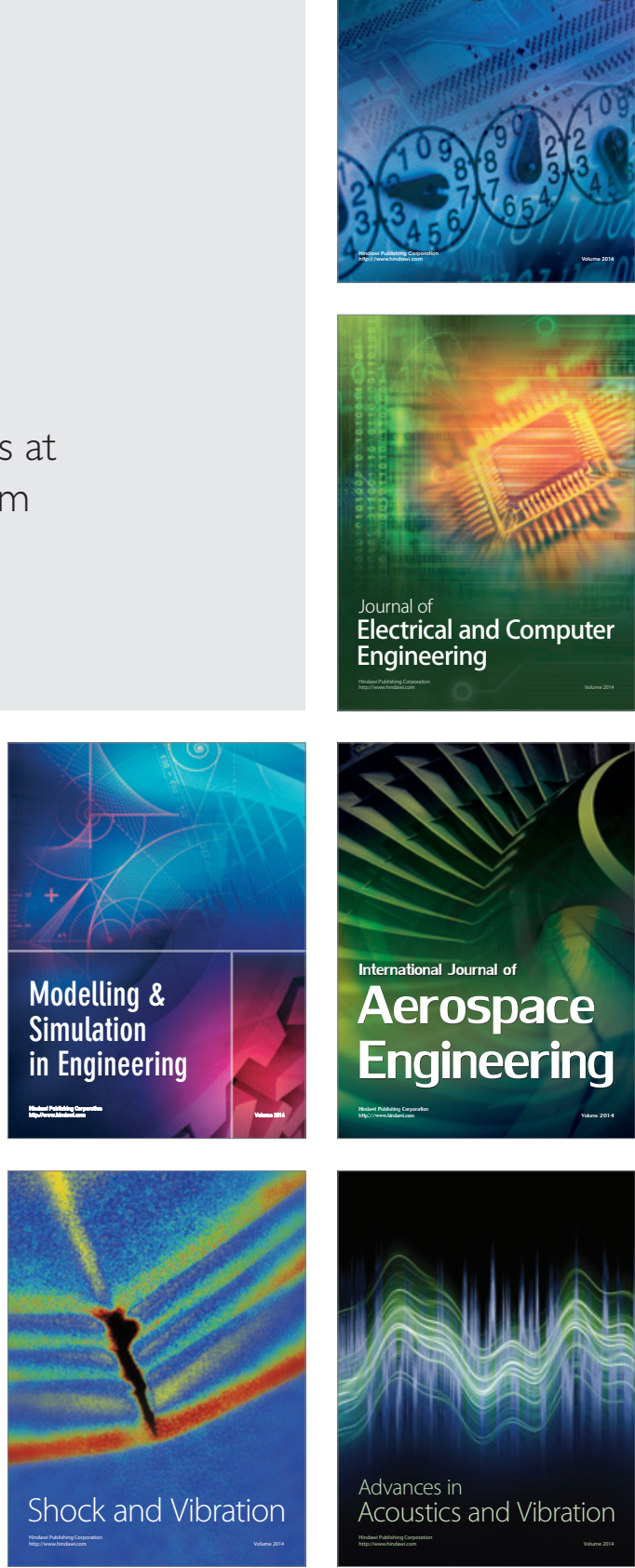